\title{
金属镇(II)配合物的合成及高选择性检测硝基化合物的 性质
}

陈敏敏，严文艳，李红喜"，郎建平*

苏州大学材料与化学化工学部, 苏州 215123

*联系人, E-mail: lihx@ @suda.edu.cn; jplang@suda.edu.cn

2015-12-21 收稿, 2016-01-25 修回, 2016-01-26 接受, 2016-03-14 网络版发表

国家自然科学基金(21373142, 21531006)资助

摘要 在室温扩散或溶剂热条件下, $\mathrm{Mn}(\mathrm{OAc})_{2} \cdot 4 \mathrm{H}_{2} \mathrm{O}$ 和 $N, N^{\prime}$-二氧化- $4,4^{\prime}$-联吡啶 $(\mathrm{BPNO})$ 分别与 1,4 - 菜二酸 $\left(1,4-\mathrm{H}_{2} \mathrm{NDC}\right)$ 或 5-氨基间苯二甲酸 $\left(\mathrm{NH}_{2}-\mathrm{H}_{2} \mathrm{BDC}\right)$ 反应，分离得到 3 个配位聚合物 $\left[\mathrm{Mn}_{2}(1,4-\mathrm{NDC})_{2}(\mathrm{BPNO})_{2}\left(\mathrm{H}_{2} \mathrm{O}\right)_{6}\right.$. $\left.(\mathrm{BPNO})_{3}\right]_{n}(\mathbf{1}),\left[\mathrm{Mn}_{2}(\mathrm{BPNO})(1,4-\mathrm{NDC})_{2} \cdot \mathrm{MeOH}\right]_{n}(\mathbf{2})$ 和 $\left[\mathrm{Mn}_{2}\left(\mathrm{NH}_{2}-\mathrm{BDC}\right)_{2}(\mathrm{BPNO})(\mathrm{DMF})_{2}\right]_{n}(\mathbf{3})$. 对这些化合物进行了红 外、元素分析、热重、粉末 $X$ 线衍射、单晶X射线衍射等表征. 其中, 化合物 3 在乙腈溶液中有着较好的稳定性和 良好的荧光性质，可用来检测硝基类芳香化合物，它对检测2,4-DNP有着很高的选择性，并可循环使用.

关键词锰化合物, 配位聚合物, 晶体结构, 合成, 硝基化合物的检测

硝基类化合物作为环境污染物和潜在的爆炸物 而备受人们关注 ${ }^{[1 ~ 3]}$. 目前, 检测硝基类化合物的主 要方法依赖于受过训练的防爆犬或精密的仪器设备 如气质联用色谱仪、表面增强拉曼光谱仪等 ${ }^{[4,5]}$. 这些 检测费用昂贵、操作繁琐. 而基于苂光淬灭的探针法 具有快速、高效、灵敏、操作简单等特点 ${ }^{[6]}$. 近年来, 结构新颖多样的配位聚合物可通过调节金属离子、有 机配体、分子骨架进而调控其苂光性质, 配位聚合物 已开始应用于高效检测痕量硝基类化合物 ${ }^{[7,8]}$. 因此, 设计合成一种性质稳定、对检测硝基类化合物选择性 高的配位聚合物材料尤为重要. 本文选取 $N, N^{\prime}$-二氧 化-4,4'-联吡啶(BPNO)配体和 1,4-慕二酸或 5-氨基间 苯二甲酸 $\left(\mathrm{NH}_{2}-\mathrm{H}_{2} \mathrm{BDC}\right)$ 与 $\mathrm{Mn}(\mathrm{OAc})_{2} \cdot 4 \mathrm{H}_{2} \mathrm{O}$ 在室温扩 散或溶剂热反应条件下得到了 3 个配位聚合物 $\left[\mathrm{Mn}_{2}-\right.$ $\left.(1,4-\mathrm{NDC})_{2}(\mathrm{BPNO})_{2}\left(\mathrm{H}_{2} \mathrm{O}\right)_{6} \cdot(\mathrm{BPNO})_{3}\right]_{n}(\mathbf{1}),\left[\mathrm{Mn}_{2}(\mathrm{BPNO})\right.$ $\left.(1,4-\mathrm{NDC})_{2} \cdot \mathrm{MeOH}\right]_{n}(2)$ 和 $\left[\mathrm{Mn}_{2}\left(\mathrm{NH}_{2}-\mathrm{BDC}\right)_{2}(\mathrm{BPNO})\right.$ $\left.(\mathrm{DMF})_{2}\right]_{n}(\mathbf{3})$. 其中化合物 $\mathbf{3}$ 在乙腈中有着较好的稳定
性和良好的苂光性质，可高效地检测乙腈中痕量硝 基类化合物。

\section{1 实验}

(i ) 仪器与试剂. 配体BPNO按文献[9]方法合 成, 其他试剂均为分析纯试剂, 未经进一步纯化. 元 素分析数据在EA1110-CHNO-S型Carlo-Erba元素分 析仪测定; 红外光谱在 Varian 1000型FT-IR光谱仪 (400 4000 $\left.\mathrm{cm}^{-1}\right), \mathrm{KBr}$ 压片获得; PXRD是利用PANalytical X'Pert PRO MPD(PW3040/60) X射线多晶衍射 仪分析测试后得到; 热稳定性由Perkin-Elmer TGA-7 热重分析仪在升温速率 $10^{\circ} \mathrm{C} / \mathrm{min}$, 空气流量为 100 $\mathrm{cm}^{3} / \mathrm{min}$ 的情况下测得; 苂光光谱在 Perkin-Elmer LS55苂光分光光度计上或者是在Varian Cary Elipse 苂光分光光度计上测得。

(ii) 配合物的合成. 配合物 $\mathbf{1}$ 的合成:将BPNO $(28 \mathrm{mg}, 0.15 \mathrm{mmol})$ 和 1,4 -芸二酸 $(32 \mathrm{mg}, 0.15 \mathrm{mmol})$ 
溶于 $1 \mathrm{~mL} N, N$-二甲基甲酰胺(DMF)和 $0.1 \mathrm{~mL}$ 稀 $\mathrm{HNO}_{3}(0.1 \mathrm{~mol} / \mathrm{L})$, 并置于试管底部, 缓冲层用 $0.5 \mathrm{~mL}$ $\mathrm{DMF}$; 然后再缓慢滴加 $\mathrm{Mn}(\mathrm{OAc})_{2} \cdot 4 \mathrm{H}_{2} \mathrm{O}(50 \mathrm{mg}, 0.2$ $\mathrm{mmol})$ 的DMF溶液, 上层用 $10 \mathrm{~mL}$ 的无水乙醚覆盖, 密封静置一周, 得到粉红色的块状晶体 1 , 过滤收集 晶体, 用乙醚洗涤, 空气中干燥. 产率: $28 \mathrm{mg}, 60 \%$ (由 BPNO计算得到); 元素分析 $\left(\mathrm{C}_{37} \mathrm{H}_{32} \mathrm{~N}_{5} \mathrm{O}_{12} \mathrm{Mn}\right)$, 理 论值(\%): C, 56.00; H, 4.06; N, 8.82; 实测值(\%): C, 55.12; H, 4.03; N, 8.77. IR(KBr压片, $\left.\mathrm{cm}^{-1}\right)$ : 1562(s), 1472(s), 1405(m), 1384(w), 1364(m), 1244(s), 1175 (m), 1026(m), 839(m), 800(m), 755(w), 549(m), 537 (m), 511(m).

配合物 2 的合成: 将BPNO(28 mg, $0.15 \mathrm{mmol})$ 、 1,4- $\mathrm{H}_{2} \mathrm{NDC}(32 \mathrm{mg}, 0.15 \mathrm{mmol})$ 和 $\mathrm{Mn}(\mathrm{OAc})_{2} \cdot 4 \mathrm{H}_{2} \mathrm{O}(50$ $\mathrm{mg}, 0.2 \mathrm{mmol}) 、 2 \mathrm{~mL} \mathrm{MeOH}$ 和稀 $\mathrm{HNO}_{3}(0.1 \mathrm{~mol} / \mathrm{L}, 0.1$ $\mathrm{mL}$ ) 加人耐热的玻璃管中, 密封后放人烘箱中, 在 $120^{\circ} \mathrm{C}$ 下反应 $16 \mathrm{~h}$ 后以每 $5^{\circ} \mathrm{C} / \mathrm{h}$ 的速度缓慢降温至室 温. 得到橙色针状晶体 2 , 过滤收集晶体, 用乙醚洗 涤, 空气中干燥. 产率: $48 \mathrm{mg}, 85 \%$ (由 $1,4-\mathrm{H}_{2} \mathrm{NDC}$ 计 算得到); 元素分析 $\left(\mathrm{C}_{35} \mathrm{H}_{24} \mathrm{~N}_{2} \mathrm{O}_{11} \mathrm{Mn}_{2}\right)$ : 理论值(\%): $\mathrm{C}$, 55.43; H, 3.19; N, 3.69; 实测值(\%): C, 55.32; H, 3.13; $\mathrm{N}, 3.75 . \operatorname{IR}\left(\mathrm{KBr}\right.$ 压片, $\left.\mathrm{cm}^{-1}\right)$ : 3398(m), 1561(s), 1509(w), 1474(m), 1460(s), 1408(m), 1385(w), 1367(s), 1260 (w), 1215(m), 1182(m), 836(m), 792(m), 591(w), 570 (w), 565(w).

配合物 $\mathbf{3}$ 的合成: 3 的合成方法与 $\mathbf{2}$ 类似, 只是把 1,4- $\mathrm{H}_{2} \mathrm{NDC}$ 换成 $\mathrm{NH}_{2}-\mathrm{H}_{2} \mathrm{BDC}(36 \mathrm{mg}, 0.2 \mathrm{mmol}$ )并在稀 $\mathrm{NaOH}(0.1 \mathrm{~mol} / \mathrm{L}, 0.1 \mathrm{~mL})$ 和 $2 \mathrm{~mL} \mathrm{DMF}$ 条件下反应. 产率: $26 \mathrm{mg}, 32 \%$ (由 BPNO计算得到); 元素分析 $\left(\mathrm{C}_{16} \mathrm{H}_{15} \mathrm{~N}_{3} \mathrm{O}_{6} \mathrm{Mn}\right)$ : 理论值 $(\%): \mathrm{C}, 48.01 ; \mathrm{H}, 3.78 ; \mathrm{N}$, 10.50 ; 实测值 $(\%)$ ： C $48.12 ; \mathrm{H}, 3.63 ; \mathrm{N}, 10.45$. $\mathrm{IR}\left(\mathrm{KBr}\right.$ 压片, $\left.\mathrm{cm}^{-1}\right)$ : 3433(s), 3345(s), 1674(s), 1572(m), 1540 (m), 1472(m), 1429(m), 1384(s), 1325(w), 1220(m), 1177(m), 1108(m), 1033(w), 833(m), 789(m), 725(m), 672(m), 550(m).

(iii) 晶体结构的测定. 衍射使用的晶体直接从 上面合成的晶体中挑选. 选用大小合适的晶体在 Bruker APEX-II CC(1), Agilent Xcalibur(2)或Rigaku Mercury CCD X-ray (3) 衍射仪上收集数据, 采用经石 墨单色器单色化的Mo K $\alpha$ 辐射 $(\lambda=0.71070 \AA)$, 液氮冷 却到 $223 \mathrm{~K}(\mathbf{1}$ 和 3) 或 $262 \mathrm{~K}(\mathbf{2}), \omega$ 扫描模式. 用 CrysAlisPro(Agilent Technologies, Ver. 1.171.35.21, 2012)和Bruker SAINT对基于可观测点获得的晶胞参
数进行精修, 用Crystalstructure(Rigaku and MSC, version 3.60,2004)程序还原, 衍射数据经 $L p$ 因子和 吸收校正, 均用直接法(SHELXS-97)解出 ${ }^{[10,11]}$, 随后 用数轮差值Fourier合成法确定了全部非氢原子的坐 标, 继而基于 $F^{2}$ 用全矩阵最小二乘法对所有非氢原 子进行各向异性温度因子修正.

氢原子坐标均通过理论方法加人, 并采用各向 同性温度因子进行修正. 其键长为 $\mathrm{C}-\mathrm{H}=0.98 \AA$ (亚甲 基), $\mathrm{C}-\mathrm{H}=0.97 \AA$ (甲基), $\mathrm{C}-\mathrm{H}=0.94 \AA$ (芳环), 热因子 为 $U_{\mathrm{iso}}(\mathrm{H})=1.2 U_{\mathrm{eq}}(\mathrm{C})$ (亚甲基), $U_{\mathrm{iso}}(\mathrm{H})=1.5 U_{\mathrm{eq}}(\mathrm{C})$ (甲 基), $U_{\mathrm{iso}}(\mathrm{H})=1.2 U_{\mathrm{eq}}(\mathrm{C})$ (芳环). 所有计算均采用 SHELXTL-97程序包在PC机上进行. 1中的水O9原子 和 2 中甲醇 $\mathrm{O} 11$ 原子没有加氢. 1 3 相关的结晶学数据 和精修结果列于表 $S 1$ 中. 部分键长键角数据列于表 S2中. 其晶体学信息文件可以从剑桥晶体学数据库 (www.ccdc.cam.ac.uk/data_request/cif) 获得, 号码为 CCDC 1441931-1441933.

\section{2 结果与讨论}

\section{1 合成讨论}

在稀 $\mathrm{HNO}_{3}$ 存在的条件下, $\mathrm{Mn}(\mathrm{OAc})_{2} \cdot 4 \mathrm{H}_{2} \mathrm{O}$ 与 BPNO和 1,4- $\mathrm{H}_{2} \mathrm{NDC}$ 在DMF中室温扩散反应, 分离得 到粉红色晶体 1 , 产率为 $60 \%$. 而 $\mathrm{Mn}(\mathrm{OAc})_{2} \cdot 4 \mathrm{H}_{2} \mathrm{O}$ 与 BPNO 和 1,4- $\mathrm{H}_{2} \mathrm{NDC}$ 在甲醇中溶剂热反应, 高产率 (85\%)地得到橙色晶体 2 . 由此可见, 反应温度对于 $\mathbf{1}$ 和 $\mathbf{2}$ 的形成和结构起着决定性作用. 与 $\mathbf{2}$ 同样的反应 条件下, 将 1,4- $\mathrm{H}_{2} \mathrm{NDC}$ 改为 $\mathrm{NH}_{2}-\mathrm{H}_{2} \mathrm{BDC}$, 与 $\mathrm{Mn}$ $(\mathrm{OAc})_{2} \cdot 4 \mathrm{H}_{2} \mathrm{O}$ 和 BPNO在DMF中溶剂热反应, 得到红 色晶体 3 , 产率只有 $32 \%$. 尝试了更高的温度(如 $170^{\circ} \mathrm{C}$ ) 以及不同的 $\mathrm{pH}$, 都不能提高 3 的产率.

化合物 1 3 均对空气稳定, 不溶于常见的有机溶 剂如乙腈、甲醇、四氢呋喃、甲苯和二氯甲烷. 其元 素分析与化学式相符. 在 1 3 的红外光谱中, 1385或 $1384 \mathrm{~cm}^{-1}$ 处中等强度的峰为 $\mathrm{BPNO}$ 分子上的 $\mathrm{N}-\mathrm{O}$ 的 伸缩振动. 在 3 中的 $3433 \mathrm{~cm}^{-1}$ 处的峰是配位 $\mathrm{NH}_{2}-\mathrm{BDC}$ 上的 $\mathrm{N}-\mathrm{H}$ 的伸缩振动. 1 3 的结构进一步通过 $\mathrm{X}$ 射线 单晶衍射得到证实. 1 3 的X射线粉末衍射图谱分别 与由单晶衍射数据模拟出的图谱一致, 说明 1 3 的宏 量样品中没有其他杂质(图S1), 样品是纯相. 热重分 析显示, 1 和 2 在 $20 \sim 120^{\circ} \mathrm{C}$ 之间分别失重 $7.5 \%$ 和 $3.8 \%$, 相当于失去晶格中配位的水分子或甲醇溶剂分子(理 
论值分别为 $6.8 \%$ 和 $4.49 \%$ )(图S2). 随着温度的升高继 续分解, 最后 1 3 的剩余率分别为 $11.1 \%, 17.8 \%$ 和 $21.3 \%$, 经计算, 最终残渣分别为 $\mathrm{MnO}_{2}$ (1) 理论值 $10.9 \% ; 3$, 理论值 $21.4 \%)$ 和 $\mathrm{MnO}(2$, 理论值 $18.7 \%)$. 1 3 分别在 $250^{\circ} \mathrm{C}(\mathbf{1}) 、 350^{\circ} \mathrm{C}(2)$ 和 $290^{\circ} \mathrm{C}(3)$ 温度内保持 晶体结构不变, 表明 1 3 的热稳定性较高.

\section{2 晶体结构}

（i ）化合物 $\mathbf{1}$ 的晶体结构. 化合物 $\mathbf{1}$ 属于三斜晶 系 $P \overline{1}$ 空间群, 其不对称单元包含半个 $\mathrm{Mn}_{2}(1,4-\mathrm{NDC})_{2}-$ $(\mathrm{BPNO})_{2}\left(\mathrm{H}_{2} \mathrm{O}\right)_{6} \cdot(\mathrm{BPNO})_{3}$ 分子. 每个 $\mathrm{Mn}$ 原子同时与 2 个 1,4-NDC中的 2 个 $\mathrm{O}$ 原子、3个水分子和 1 个 BPNO中 的 1 个 $\mathrm{O}$ 原子配位, 形成了一个扭曲的八面体配位构 型 (图 1).1,4-NDC 两端的羧酸分别连接 2 个相邻 的 $\left[\mathrm{Mn}(\mathrm{BPNO})\left(\mathrm{H}_{2} \mathrm{O}\right)_{3}\right]$ 单元, 形成一维链 $[\mathrm{Mn}(1,4-\mathrm{NDC})$ $\left.(\mathrm{BPNO})\left(\mathrm{H}_{2} \mathrm{O}\right)_{3}\right]_{n}$ (图1). 1,4-NDC配体采用 $\mu-\eta^{1}, \eta^{1}$ 配位
方式. 链与链之间又通过 $\mathrm{BPNO}$ 上苯环的 $\pi-\pi$ 作用将 游离的BPNO连接在一起, 形成三维结构(图S3).

（ii）化合物 $\mathbf{2}$ 的晶体结构. 化合物 2 属于单斜晶 系 $P 2_{1} / \mathrm{c}$ 空间群, 中心 $\mathrm{Mn}$ 原子同时与 2 个 $\mathrm{BPNO}$ 上的 2 个氧原子和 4 个 1,4 - 萗二酸上的 4 个氧原子配位, 形成 扭曲的八面体配位环境(图2(a)). 1,4-NDC配体采用 $\mu-\eta^{1}, \eta^{1}$ 配位方式. $M n(I I)$ 原子通过BPNO和 1,4-䒬二 酸配体桥连形成一维结构(图S4(a)), 链与链之间通 过BPNO连接, 形成平面二维结构(图S4(b)), 平面二 维结构又通过 1,4 -萗二酸配体桥连形成三维网络结 构(图2(b)).

（iii）化合物 $\mathbf{3}$ 的晶体结构. 化合物 $\mathbf{3}$ 属于三斜晶 系 $P$ 空间群. 在 3 中, $\mathrm{NH}_{2}$ - BDC配体采用 $\mu_{3}-\eta^{1}-\eta^{1}, \eta^{1}$ 配 位方式. 六配位的 $\mathrm{Mn}(\mathrm{II})$ 分别与 3 个来自 $\mathrm{NH}_{2}-\mathrm{BDC}$ 中 的 4 个氧原子、 1 个 $\mathrm{BPNO}$ 上的 1 个氧原子和一个来自 $\mathrm{DMF}$ 上的氧原子配位(图3(a)). [Mn(DMF)]单元通过

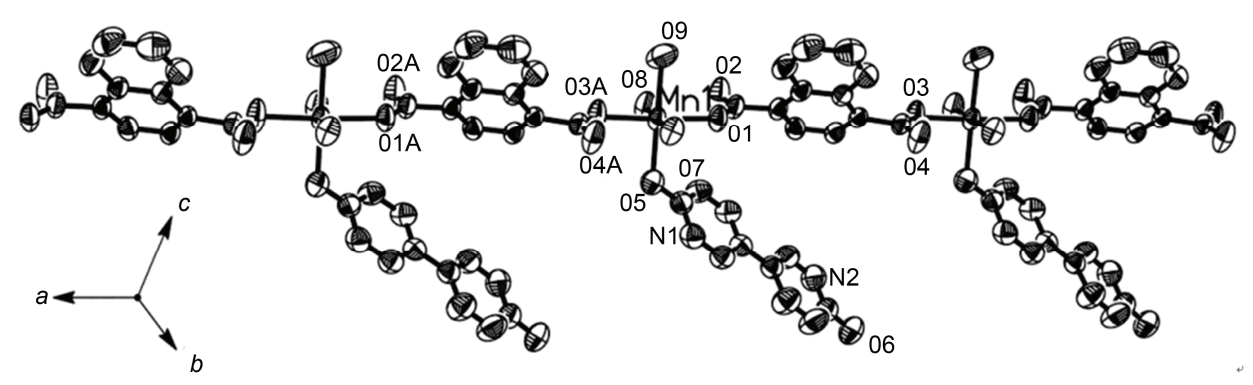

图 1 化合物 1 一维链结构. 氢原子和游离的BPNO配体未标示. 对称操作码: (A) $x+1, y, z$

Figure 1 View of a section of the chain of 1. All H atoms and BPNO ligands are omitted for clarity. Symmetry codes: (A) $x+1, y, z$
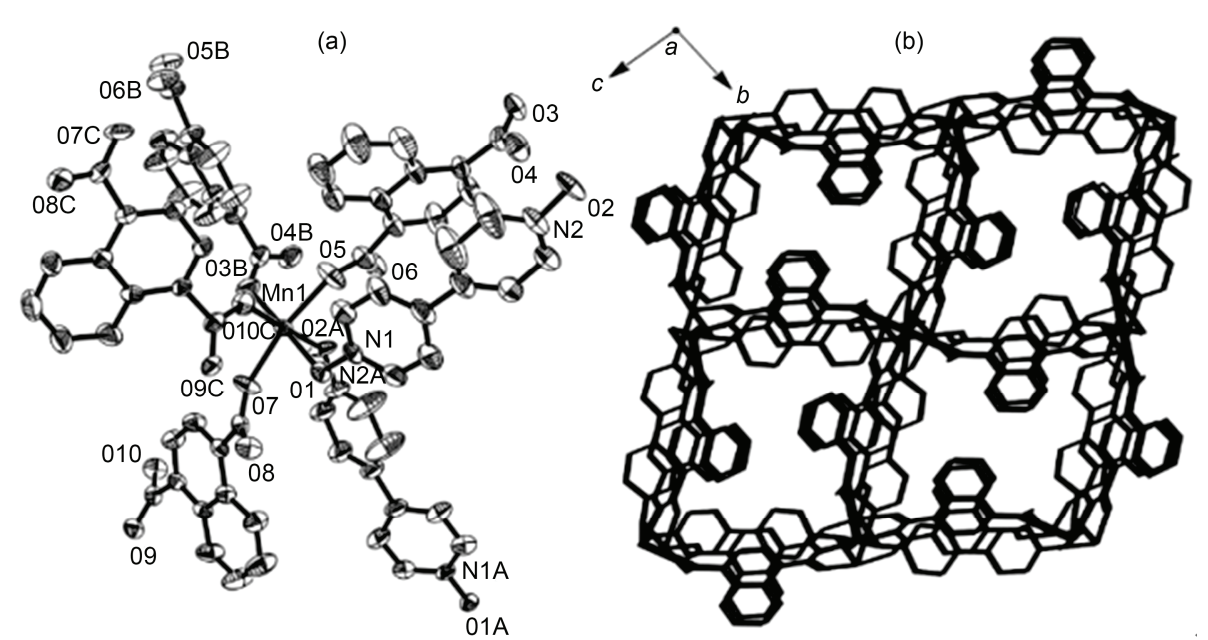

图 2 (a) 化合物 $\mathbf{2}$ 中 $\mathrm{Mn}(\mathrm{II}$ )配位环境; (b) 化合物 $\mathbf{2}$ 的三维示意图. 氢原子和游离的 $\mathrm{MeOH}$ 分子未标示. 对称操作码: (A) $x,-y+1 / 2, z-1 / 2 ;(B)$ $-x+2, y+1 / 2,-z+1 / 2 ;$ (C) $x,-y+3 / 2, z+1 / 2$

Figure 2 (a) View of the coordination environment of the $\mathrm{Mn}(\mathrm{II})$ atom in 2; (b) view of the 3D network of 2. All $\mathrm{H}$ atoms and MeOH molecules are omitted for clarity. Symmetry codes: (A) $x,-y+1 / 2, z-1 / 2$; (B) $-x+2, y+1 / 2,-z+1 / 2$; (C) $x,-y+3 / 2, z+1 / 2$ 


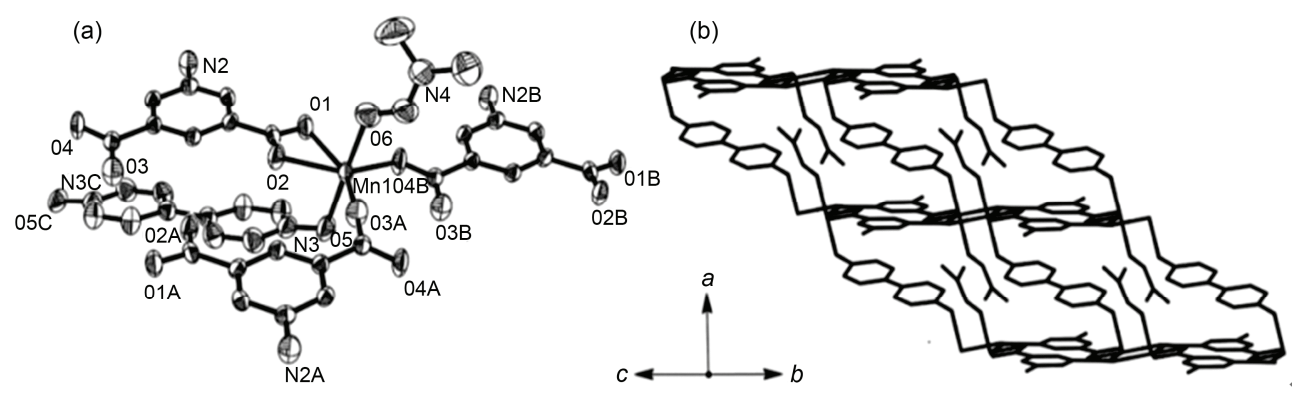

图 3 (a) 化合物 3 中 Mn(II)配位环境; (b) 化合物 $\mathbf{3}$ 的二维示意图. 氢原子未标示. 对称操作码: (A) $-x+1,-y+1,-z+1 ;$ (B) $x, y+1, z ;$ (C) $2-x, 1-y$,

$1-z$

Figure 3 (a) View of the coordination environment of the $\mathrm{Mn}(\mathrm{II})$ atom in 3; (b) view of the 2D network of 3. All $\mathrm{H}$ atoms are omitted for clarity. Symmetry codes: (A) $-x+1,-y+1,-z+1$; (B) $x, y+1, z$; (C) $2-x, 1-y, 1-z$

$\mathrm{NH}_{2}-\mathrm{BDC}$ 的羧酸桥连, 形成一维链, 链与链通过 BPNO连接, 形成二维网状结构(图3(b)).

\section{3 化合物的固体苂光性质及化合物 3 的苂光 检测硝基类芳香化合物}

如图4所示, 用波长为 $398 \mathrm{~nm}$ 光激发配体BPNO, 在472 $\mathrm{nm}$ 处发射苂光, 源于 $\pi-\pi *$ 电子跃迁 ${ }^{[12]} .1$ 和 3 分 别被波长为 327 和 $370 \mathrm{~nm}$ 的光激发, 它们均有很强的 苂光发射, 其最大发射波长分别为 467 和 $507 \mathrm{~nm}$, 而 2 并没有苂光. 1 和 3 的最强发射峰与BPNO接近, 说明 它们的苂光来源于BPNO配体. 与BPNO相比, 1 的最 强发射峰发生了略微的蓝移, 而 3 的最大发射波长发 生了红移, 这可能是源自于配体到金属间的电子跃 迁 (LMCT) 产生的影响 ${ }^{[13]}$. 本文选择 $\mathbf{3}$ 作为菼光探针 来检测硝基类芳香化合物, 分别称取 $2 \mathrm{mg}$ 的 3 分散在 的不同的有机溶剂 $(2 \mathrm{~mL}$ 腈、甲醇、四氢呋喃、甲 苯、二氯甲烷或硝基苯)中, 超声 $30 \mathrm{~min}$ 使其均匀分 散. 3 的苂光强度 $\left(\lambda_{\mathrm{ex}}=350 \mathrm{~nm}\right)$ 取决于溶剂的种类, 在 乙腈溶液中, $\boldsymbol{3}$ 的苂光强度相对较强. 有趣的是, 在硝 基苯中, 发生了苂光淬灭现象(图S5), 这是由于电子 从聚合物的激发态转移到带有吸电子基团的硝基苯 上 $^{[14]}$. 该现象和文献 ${ }^{[15]}$ 中已报道的聚合物性质很相 似, 其淬灭现象与带有吸电子的硝基基团有关. 因 此,尝试了 3 在乙腈溶液中检测硝基苯及其他硝基类 芳香化合物. 为了探究 3 对不同硝基类芳香化合物的 痕量检测, 对 4 种常见的硝基类化合物 $(2,4$-二硝基苯 酚(2,4-DNP)、对硝基苯胺、对硝基苯酚以及硝基苯) 进行了滴定实验. 如图S6所示, 将3分散在乙腈溶液 中定量加人 $150 \mathrm{ppm}(1 \mathrm{ppm}=1 \mathrm{mg} / \mathrm{L})$ 的硝基化合物, 2,4-DNP的淬灭效率是 $81 \%$, 明显高于其他硝基化合

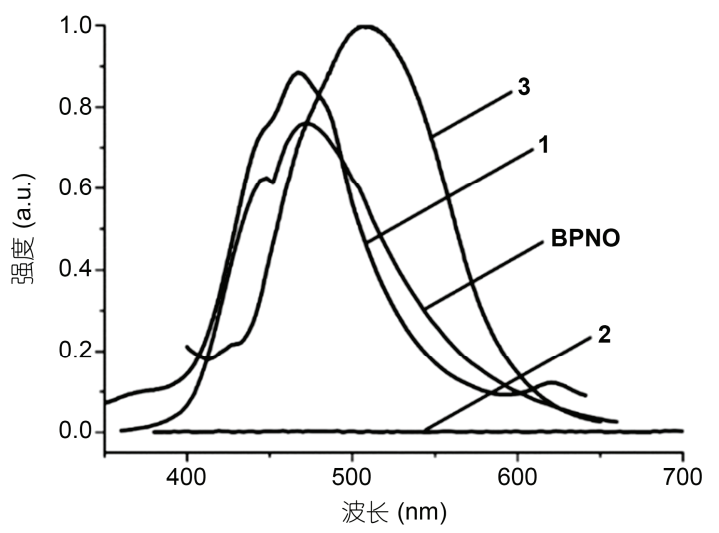

图 4 化合物 1, 2, 3 和BPNO在室温下的固体苂光光谱. 苂光强度数 据都已进行归一化处理

Figure 4 Emission spectra of 1, 2, 3 and BPNO in the solid-state at ambient temperature. The intensity of the fluorescence is normalized

物的淬灭效率(33\%(对硝基苯胺)、13\%(对硝基苯酚) 和 6\%(硝基苯)). 硝基化合物的紫外吸收光谱和 3 的 发射峰光谱的重复面积决定着能量转移的强弱 ${ }^{[16,17]}$. 由图S7可知, 2,4-DNP的紫外吸收在400 475 nm处都 能和 3 的苂光发射峰重叠, 即重复的面积多于对硝基 苯胺、对硝基苯酚以及硝基苯的面积. 因此根据实验 结果, 可知苂光淬灭效率的顺序和其覆盖面积一致.

为了进一步研究其苂光淬灭的过程, 将 $2 \mathrm{mg}$ 的 $\mathbf{3}$ 均匀分散在 $2 \mathrm{~mL}$ 的 $\mathrm{MeCN}$ 溶剂中, 利用 4 种 $5 \times 10^{-3}$ $\mathrm{mmol} \mathrm{L} \mathrm{L}^{-1}$ 的硝基类化合物对其进行滴定, 得到了溶 液苂光强度与硝基类化合物浓度的关系图(图 S8), 以 $\lambda_{\mathrm{em}}=428 \mathrm{~nm}$ 处苂光强度与硝基化合物的浓度关系, 得到的Stern-Volmer(SV)点图(图5(a)). 通过SV方程 $\left(I_{0} / I\right)=K_{\mathrm{sv}}[A]+1$ (其中 $I_{0}$ 和 $I$ 分别是加人分析物之前和之 后的苂光强度, $K_{\mathrm{sv}}$ 是淬灭常数 $\left(\mathrm{L} \mathrm{mmol}{ }^{-1}\right)$, 而 $[A]$ 则是 分析物的摩尔浓度), 可以算出淬灭常数, 进而可以 

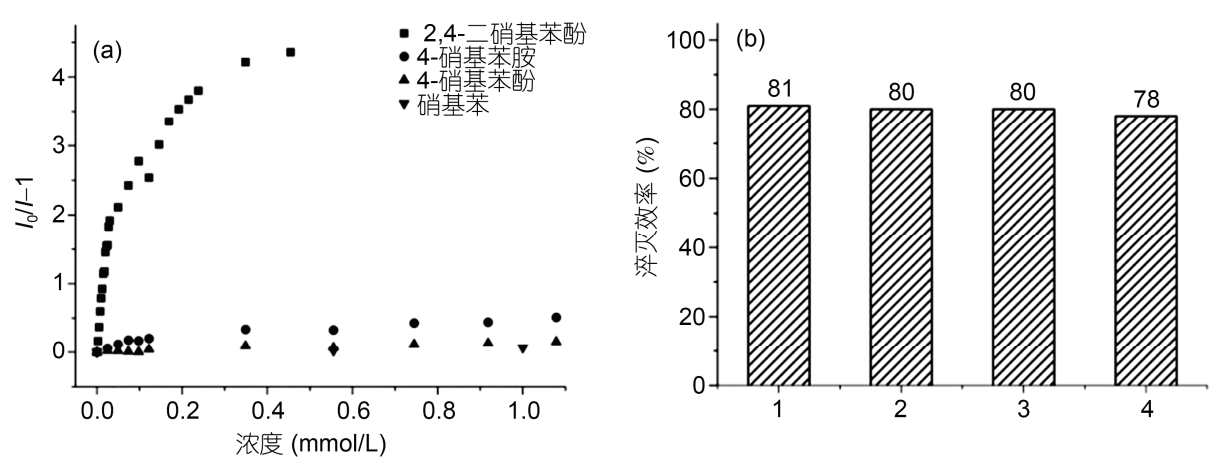

图 5 (a) 化合物 $\mathbf{3}$ 分散在乙腈中加人不同硝基化合物的Stern-Volmer点图; (b) 化合物 $\mathbf{3}$ 分散在乙腈中加人 150 ppm的 2,4-二硝基苯酚后的菼 光淬灭循环图

Figure 5 (a) Stern-Volmer (SV) plots of $\mathbf{3}$ for various nitro derivatives in MeCN; (b) recyclability of $\mathbf{3}$ dispersed in MeCN in the presence of 150 ppm 2,4-DNP

分析其淬灭效率. 由图 5(a)可知, 2,4-二硝基苯酚、对 硝基苯胺、对硝基苯酚以及硝基苯的淬灭常数分别是 9.72, 0.399, 0.137和 $0.0644 \mathrm{~L} / \mathrm{mmol} .2$,4-DNP的淬灭 常数明显高于其他 3 个硝基化合物, 其苂光淬灭效率 也是最高的. 由此可见, 3对 2,4-DNP有着很高的选择 性. 从 SV点图可知, 在低浓度时, 2,4-DNP的点图基本 呈现线性关系, 而随着加人 2,4-DNP的浓度增加则偏 离线性关系. 2,4-DNP的非线性SV点图, 可能是由化 合物间自我吸收或者是能量转移造成的. 以上这些结 果表明苂光淬灭同时包含动态和静态的过程 ${ }^{[16,17]}$.

本文也研究了 2,4-DNP浓度和苂光淬灭效率的关 系. 在 3 的 $\mathrm{MeCN}$ 溶液中, 定量地加人 2,4-DNP(0 83 $\mathrm{ppm}$ ). 如图S9所示, 在低浓度的2,4-DNP中, 3 具有很 高的淬灭速率, 其中 2,4-DNP的浓度在 1 6 $\mathrm{ppm}$, 呈 现较好的线性关系 $\left(y=0.0579 x+0.3469, R^{2}=0.941\right)$. 随 着2,4-DNP浓度的继续增大, 淬灭速率明显减慢; 最 后当加人 $150 \mathrm{ppm}$ 的2,4-DNP时, 淬灭效率达到 $81 \%$. 因此, 3 的苂光淬灭过程是通过能量转移和电子转移 的方式来实现的. 3对 2,4-DNP检测的选择性高、检测 限低. 3在乙腈、2,4-二硝基苯酚 (2,4-DNP)、对硝基苯 胺、对硝基苯酚以及硝基苯中浸泡 $24 \mathrm{~h}$ 后, 经PXRD 测试表明(见图 $\mathrm{S} 1$ 和 $\mathrm{S} 10$ ), X射线粉末衍射图谱与原图
谱一致, 说明化合物原来的骨架还能保持, 说明 $\mathbf{3}$ 在 以上几种溶液中有着很好的稳定性. 另外, 当把检测 过后 3 的乙腈溶液离心分离后, 可以重复使用数次, 还能保持很强的苂光强度和对 2,4-DNP的苂光淬灭效 率(图5(b)).

\section{3 结论}

本文通过室温扩散和溶剂热的方法合成了 3 个配 位聚合物 $\left[\mathrm{Mn}_{2}(1,4-\mathrm{NDC})_{2}(\mathrm{BPNO})_{2}\left(\mathrm{H}_{2} \mathrm{O}\right)_{6} \cdot(\mathrm{BPNO})_{3}\right]_{n}$ (1), $\left[\mathrm{Mn}_{2}(\mathrm{BPNO})(1,4-\mathrm{NDC})_{2} \cdot \mathrm{MeOH}\right]_{n}(\mathbf{2})$ 和 $\left[\mathrm{Mn}_{2}\left(\mathrm{NH}_{2}-\right.\right.$ $\left.\mathrm{BDC})_{2}(\mathrm{BPNO})(\mathrm{DMF})_{2}\right]_{n}(\mathbf{3})$. 在化合物 $\mathbf{1}$ 中, $[\mathrm{Mn}$ $\left.(\mathrm{BPNO})\left(\mathrm{H}_{2} \mathrm{O}\right)_{3}\right]$ 单元通过 1,4-NDC桥连, 形成一维聚 合链. 在化合物 2 中, $\mathrm{Mn}$ 原子通过一对 $\mathrm{BPNO}$ 和 4 个 1,4-萗二酸桥连, 形成三维结构. 在化合物 3 中, $\mathrm{NH}_{2}-$ $\mathrm{BDC}$ 和BPNO连接 $[\mathrm{Mn}(\mathrm{DMF})]$ 单元形成了一个二维网 状结构. 化合物 $\mathbf{3}$ 在乙腈溶液中有着较好的稳定性和 良好的菼光性质. 在乙腈溶液中, 化合物 $\mathbf{3}$ 利用苂光 淬灭检测 4种常见硝基类芳香化合物：2,4-二硝基苯 酚(2,4-DNP)、对硝基苯胺、对硝基苯酚以及硝基苯. 实验结果表明，化合物 $\mathbf{3}$ 作为稳定的聚合物材料对 2,4-DNP有着很高的选择性并能重复利用，因此可以 很好地被用于硝基类芳香化合物的检测.

\section{参考文献}

1 McQuade D T, Pullen A E, Swager T M. Conjugated polymer-based chemical sensors. Chem Rev, 2000, 100: 2537-2574

2 Toal S J, Trogler W C. Polymer sensors for nitroaromatic explosives detection. J Mater Chem, 2006, 16: 2871-2883

3 Germain M E, Knapp M J. Optical explosives detection: From color changes to fluorescence turn-on. Chem Soc Rev, 2009, 38: $2543-2555$ 
4 Moore D S. Instrumentation for trace detection of high explosives. Rev Sci Instrum, 2004, 75: 2499-2512

5 Czarnik A W. A sense for landmines. Nature, 1998, 394: 417-418

6 Xin Y, Wang Q, Liu T, et al. A portable and autonomous multichannel fluorescence detector for on-line and in situ explosive detection in aqueous phase. Lab Chip, 2012, 12: 4821-4828

7 Shi Z Q, Guo Z J, Zheng H G. Two luminescent Zn(II) metal-organic frameworks for exceptionally selective detection of picric acid explosives. Chem Commun, 2015, 51: 8300-8303

8 Cui Y, Yue Y, Qian G, et al. Luminescent functional metal-organic frameworks. Chem Rev, 2012, 112: 1126-1162

9 Lu W J, Zhang L P, Song H B, et al. Novel lanthanide(III) coordination networks based on 1,2-bis (4-pyridyl) ethane- $N, N$ '-dioxide and trans-1,2-bis(4-pyridyl) ethene- $N, N$ '-dioxide. New J Chem, 2002, 26: 775-781

10 Sheldrick G M. SHELXS-97, Program for Solution of Crystal Structures. Germany: University of Göttingen, 1997

11 Sheldrick G M. SHELXL-97, Program for Refinement of Crystal Structures. Germany: University of Göttingen, 1997

12 Lee SY, Park S, Kim H J, et al. Ligand-and anion-directed assembly of exo-coordinated mercury(II) halide complexes with $\mathrm{O}_{2} \mathrm{~S}_{2}$-donor macrocycles. Inorg Chem, 2008, 47: 1913-1915

13 Li S L, Lan Y Q, Ma J F, et al. Structures and luminescent properties of seven coordination polymers of zinc(II) and cadmium(II) with 3,3',4,4'-benzophenone tetracarboxylate anion and bis(imidazole). Cryst Growth Des, 2008, 8: 675-684

14 Zheng Q, Yang F, Deng M, et al. A porous metal-organic framework constructed from carboxylate-pyrazolate shared heptanuclear zinc clusters: Synthesis, gas adsorption, and guest-dependent luminescent properties. Inorg Chem, 2013, 52: 10368-10374

15 Chen M M, Zhou X, Li H X, et al. A luminescent 2D coordination polymer for selective and recyclable sensing of nitroaromatic compounds with high sensitivity in water. Cryst Growth Des, 2015, 15: 2753-2760

16 Nagarkar S S, Joarder B, Chaudhari A K, et al. Highly selective detection of nitro explosives by a luminescent metal-organic framework. Angew Chem Int Ed, 2013, 52: 2881-2885

17 Ramachandra S, Popovic Z D, Schuermann K C, et al. Förster resonance energy transfer in quantum dot-dye-loaded zeolite L nanoassemblies. Small, 2011, 7: 1488-1494

\section{补充材料}

表S1 化合物 $1 \sim 3$ 的晶体数据和结构精修参数

表S2 化合物 $1 \sim 3$ 的部分键长 $(\AA)$ 和键角 $\left({ }^{\circ}\right)$

图S1 化合物的粉末X射线衍射图

图S2 化合物 1 3 的热重分析图

图S3 化合物 1 的三维示意图

图S4 化合物2的一维链结构和二维示意图

图S5 化合物 3 分散在各种溶剂中的苂光光谱

图S6 化合物 3 (分散在乙腈)中加入 $150 \mathrm{ppm}$ 的硝基化合物的苂光淬灭效率

图S7 硝基化合物 $\left(5 \times 10^{-2} \mathrm{mmol} \mathrm{L}^{-1}\right)$ 在乙腈中的紫外吸收光谱和 $2 \mathrm{mg}$ 化合物 3 分散在 $2 \mathrm{~mL}$ 腈中的苂光发射的谱图

图S8 化合物3(分散在乙腈)中加人不同浓度的硝基化合物的荧光发射光谱图

图S9 化合物 3 (分散在乙腈)中加人不同浓度的2,4-二硝基苯酚的淬灭效率点图

图S10 化合物3分别浸泡在4-硝基苯胺、4-硝基苯酚和硝基苯 $24 \mathrm{~h}$ 后的粉末X射线衍射图

本文以上补充材料见网络版 csb.scichina.com. 补充材料为作者提供的原始数据, 作者对其学术质量和内容负责. 


\title{
Coordination polymers based on Mn(II) and BPNO with selective and recyclable sensing of nitroaromatics
}

\author{
CHEN MinMin, YAN WenYan, LI HongXi \& LANG JianPing \\ College of Chemistry, Chemical Engineering and Materials Science, Soochow University, Suzhou 215123, China
}

Detection of nitroaromatic compounds as both potential explosives and environmental pollutants is important for national homeland security, environmental protection or military applications. Current detection methods are based on some sophisticated instruments including energy dispersive X-ray diffraction, gas chromatography coupled with mass spectrometry, nuclear quadrupole resonance, surface enhanced Raman spectroscopy, neutron activation analysis, electron capture detection, and cyclic voltammetry. These instruments are very expensive and not easily accessible in most conditions. As a simpler, quicker and less expensive method, the sensitive optical detection could potentially be widely employed. The coordination polymers (CPs) could be efficiently modulated by both analyte-metal and analyte-ligand interactions. Thus the CP-based fluorescent detection of nitro-explosives displays fast, sensitive and reversible compared with other methods. It is necessary to rationally design and synthesize appropriate ligands to prepare the stable and bifunctional CPs. In this work, three $\mathrm{Mn}(\mathrm{II})$ coordination polymers $\left[\mathrm{Mn}_{2}(1,4-\mathrm{NDC})_{2}(\mathrm{BPNO})_{2}\left(\mathrm{H}_{2} \mathrm{O}\right)_{6} \cdot(\mathrm{BPNO})_{3}\right]_{n}(\mathbf{1}$, 1,4- $\mathrm{H}_{2} \mathrm{NDC}=1$,4-naphthalenedicarboxylic acid, BPNO=1,2-Bis(4-pyridyl)- $N, N^{\prime}$-dioxide), $\left[\mathrm{Mn}_{2}(\mathrm{BPNO})(1,4-\mathrm{NDC})_{2}\right.$. $\mathrm{MeOH}]_{n}$ (2) and $\left[\mathrm{Mn}_{2}\left(\mathrm{NH}_{2}-\mathrm{BDC}\right)_{2}(\mathrm{BPNO})(\mathrm{DMF})_{2}\right]_{n}\left(\mathbf{3}, \mathrm{NH}_{2}-\mathrm{H}_{2} \mathrm{BDC}=5\right.$-aminoisophthalic acid) have been synthesized by the diffusion or solvothermal reactions of $\mathrm{Mn}$ (II) salt with $\mathrm{BPNO}$ and $1,4-\mathrm{H}_{2} \mathrm{NDC}$ or $\mathrm{NH}_{2}-\mathrm{H}_{2} \mathrm{BDC}$. Complexes 1-3 were characterized by IR, elemental analysis, thermogravimetric analysis (TGA), single crystal X-ray diffraction and powder X-ray diffraction (PXRD). For compound 1, the $\left[\mathrm{Mn}(\mathrm{BPNO})\left(\mathrm{H}_{2} \mathrm{O}\right)_{3}\right]$ unit is connected by 1,4-NDC ligands to yield a 1D chain. For compound 2, a Mn(II) center is bridged by two BPNO and four 1,4-NDC ligands to generate a 3D framework. For compound 3, the [Mn(DMF)] unit is connected by $\mathrm{NH}_{2}-\mathrm{BDC}$ and BPNO ligands to form a 2D network. Among them, compound $\mathbf{3}$ shows relatively strong emission at $507 \mathrm{~nm}$ in the solid state when excited at $370 \mathrm{~nm}$ at ambient temperature. A dispersed suspension of compound $\mathbf{3}$ in $\mathrm{MeCN}$ also exhibits relatively strong luminescent emission. This emission can be selectively quenched by nitroaromatics in $\mathrm{MeCN}$ with good sensitivity. We choose compound 3 to detect 2,4-DNP, 4-nitroaniline, 4-nitrophenol and nitrobenzene in MeCN. The results show that compound $\mathbf{3}$ possesses higher selective and recyclable sensing of 2,4-DNP among four nitroaromatics and could be a good candidate for the detection of nitroaromatics.

Mn(II), coordination polymers, crystal structure, synthesis, detection of nitroaromatics

doi: 10.1360/N972015-01410 